\title{
Validação de livro educativo: Tecnologia educacional para o ensino de feridas
}

\author{
Validation of an educational book: Educational technology for teaching wounds \\ Validación de un libro educativo: Tecnología educativa para la enseñanza de las heridas
}

\section{Resumo}

Objetivo: construir e validar um livro sobre ensino de feridas, classificação, avalição e tratamento de feridas, a fim de torná-lo uma tecnologia educacional em saúde para ser utilizada por estudantes e profissionais da saúde. Metodologia: Trata-se de um desenvolvimento metodológico para validação de um livro sobre ferida. A amostra do estudo foi composta por juízes especialistas, necessário no processo de validação pela importância do julgamento do conteúdo específico e temático do livro. Para a análise quantitativa dos dados, foi realizado o cálculo do Índice de Validade de Conteúdo (IVC) e para avaliar o questionário usado nesse estudo foi utilizado o coeficiente de alfa de Cronbach. Resultados: O instrumento foi considerado validado pelos juízes, demonstrando consistência interna desejável com coeficiente alfa de Cronbach de 0,95 o qual se trata de um importante instrumento quando se objetiva medir construto único por meio de múltiplos itens, sendo que o livro apresentou IVC global de 0,99 com excelente nível de concordância, podendo-se inferir que a ferramenta é representativa do conteúdo a ser abordado sobre o ensino de enfermagem em relação à ferida. Conclusão: o livro configurando-se em uma tecnologia educacional válida para ser usada no ensino de avaliação e tratamento de feridas no ensino de enfermagem, a fim de proporcionar um processo de aprendizagem significativo e efetivo para os discentes.

Palavras-chave: Tecnologia educacional; Feridas; Educação em saúde; Enfermagem; Estudo de validação.

\begin{abstract}
Purpose: To construct and validate a book on wound teaching, classification, assessment and treatment of wounds in order to make it a health educational technology to be used by students and health professionals. Methodology: This is a methodological development for validation of a wound book. The study sample consisted of expert judges, which was necessary in the validation process because of the importance of judging the specific and thematic content of the book. For quantitative data analysis, the calculation of the Content Validity Index (CVI) was performed and to evaluate the questionnaire used in this study, Cronbach's alpha coefficient was used. Results: The instrument was
\end{abstract}


considered validated by the judges, demonstrating desirable internal consistency with Cronbach's alpha coefficient of 0.95 which is an important instrument when aiming to measure single construct through multiple items, and the book showed overall CVI of 0.99 with excellent level of agreement, it can be inferred that the tool is representative of the content to be addressed on nursing education in relation to the wound. Conclusion: the book is a valid educational technology to be used in the teaching of wound assessment and treatment in nursing education, in order to provide a meaningful and effective learning process for students.

Keywords: Educational technology; Wounds; Health education; Nursing; Validation study.

\begin{abstract}
Resumen
Objetivo: Construir y validar un libro sobre la enseñanza, la clasificación, la evaluación y el tratamiento de las heridas con el fin de convertirlo en una tecnología educativa sanitaria para ser utilizada por estudiantes y profesionales de la salud. Metodología: Se trata de un desarrollo metodológico para la validación de un libro sobre la ferida. La encuesta está compuesta por expertos en la materia, necesarios en el proceso de evaluación por la importancia del contenido específico y temático del libro. Para el análisis de los datos cuantitativos, se realizó el cálculo del Índice de Validez de Contenido (IVC) y para evaluar el cuestionario utilizado en este estudio se utilizó el coeficiente alfa de Cronbach. Resultados: El instrumento se consideró validado por los jueces, demostrando una consistencia interna deseable con un coeficiente alfa de Cronbach de 0,95 que es un instrumento importante cuando se pretende medir un solo constructo a través de múltiples ítems, y el libro mostró un CVI global de 0,99 con un excelente nivel de acuerdo, se puede inferir que la herramienta es representativa del contenido que se debe abordar en la educación de enfermería en relación con la herida. Conclusión: el libro se configura como una tecnología educativa válida para ser utilizada en la enseñanza de la evaluación y el tratamiento de las enfermedades en la enseñanza de la enfermería, con el fin de proporcionar un proceso de aprendizaje significativo y eficaz para los discentes.
\end{abstract}

Palabras clave: Tecnología educativa; Heridas; Educación sanitaria; Enfermería; Estudio de validación.

\title{
1. Introdução
}

As lesões configuram-se como um grave problema de saúde pública e estima-se que cerca de $3 \%$ da população brasileira possui alguma lesão(Melo et al., 2020). As lesões nada mais são do que uma condição em que há a ruptura da integridade da pele ou mucosa, independentemente de qual seja sua etiologia(Capella et al., 2016). Por sua vez, o tratamento de feridas concerne em um processo que requer diversas mudanças e repostas fisiológicas, muitas vezes, difíceis de se alcançar, pois exigem conhecimento e pensamento crítico para subsidiar uma sistemática avaliação e tomada de decisão (Silva \& Oliveira-Kumakura, 2018).

Portanto, o processo cicatricial depende de cuidados complexos e, geralmente, prolongados (Melo et al., 2020; Salome, 2020). Dentre os profissionais habilitados para tal tarefa, o enfermeiro ocupa lugar de destaque, uma vez que, a ele é incumbido atribuições decisivas para o sucesso do tratamento. Deste modo, é imprescindível que ele seja munido de conhecimento técnico-cientifico que permita uma avaliação acurada, a qual irá nortear o processo cicatricial(Salome, 2020). No entanto, estudos indicam que há lacunas no conhecimento dos profissionais de enfermagem no que tange o assunto, e, que tal falha, está no processo de formação dos mesmos (Silva \& Oliveira-Kumakura, 2018).

Para auxiliar na resolução de tal problemática, as tecnologias educacionais configuram-se em ótimas ferramentas quando aplicadas dentro do processo ensino-aprendizagem na graduação (Gonçalves et al., 2015; Silva \& Oliveira-Kumakura, 2018), uma vez que, configuram-se em ferramentas utilizadas para otimizar o processo de aprendizagem, pois sua finalidade é proporcionar a participação ativa dos sujeitos no processo educativo, acarretando na produção e aplicação do conhecimento, autonomia e empoderamento sobre processos e produtos e transformar a utilização empírica do conhecimento em algo científico (Carvalho et al., 2019; Wild et al., 2019).

Dentre as tecnologias educacionais pode-se citar os protocolos, manuais, cartilhas, algoritmos, guias e diretrizes (Salome, 2020; Wild et al., 2019). Além dessas tecnologias, pode se fazer uso de metodologias ativas de ensino como auxiliadoras do processo de aprendizagem, já que, elas facilitam o processo de aprendizagem do estudante. Dentre as essas, há a simulação clínica, essa metodologia de ensino é amplamente utilizada, já que, oferece uma aprendizagem de forma ativa, 
elaboração de conhecimento, desenvolvimento do senso crítico diante de uma realidade e facilita a obtenção de habilidades técnicas, como raciocínio crítico e tomada de decisão (Kumakura et al., 2018; Mazzo et al., 2017).

No que tange a avaliação e o tratamento de feridas, a simulação clínica pode ser uma estratégia eficaz dentro da graduação de enfermagem, pois é um método que incentiva os alunos desenvolverem uma relação entre a teoria e a prática, raciocínio clínico e promover uma autoconfiança na assistência ao paciente portador de feridas (Mazzo et al., 2017; Silva \& Oliveira-Kumakura, 2018).

Em relação a simulação com feridas, uma técnica que vem sendo muito utilizada em simulações (Kumakura et al., 2018; Mazzo et al., 2017), para a simulação de feridas, a qual tem-se demonstrada como uma técnica efetiva no processo de aprendizagem dos discentes é a técnica de moulage. Essa técnica utiliza materiais de maquiagem, adorno, artefatos e outros itens para conferir características clínicas de feridas, conferindo fidelidade a simulação, ou cenário, o que ativa o sistema de sensopercepção dos participantes daquele evento.

Dessa forma, é cada vez mais comum a produção de protocolos e algoritmos para auxiliar a tomada de decisão dos profissionais da saúde e direcionar o tratamento de feridas (Lima et al., 2020).

As tecnologias educacionais em saúde podem ser construídas por meio de material, instrumento, produto e/ou processo utilizado em alguma ação educativa, desde que seja, submetido a um sistemático processo de construção e validação do produto(Wild et al., 2019). Essa etapa é fundamental, pois é por meio desse processo que será conferido a certificação do conteúdo divulgado, sendo assegurado, por avaliadores, a eficácia do material disponibilizado para o uso (F. O. de A. M. da Cruz et al., 2020).

Diante do exposto, considerando a necessidade da criação de tecnologias educacionais voltadas para o ensino de feridas para estudantes e profissionais da saúde o objetivo desse estudo visa construir e validar um livro sobre ensino de feridas, classificação, avalição e tratamento de feridas, a fim de torná-lo uma tecnologia educacional em saúde para ser utilizada por estudantes e profissionais da saúde.

\section{Metodologia}

Trata-se de um desenvolvimento metodológico para validação de um livro sobre ferida. A amostra do estudo foi composta por juízes especialistas, necessário no processo de validação pela importância do julgamento do conteúdo específico e temático do livro. O levantamento dos juízes e a coleta de dados foram realizados de maio a junho de 2020.

Para a seleção dos juízes, foram estabelecidos quatro critérios de inclusão: experiência com a temática do estudo; docente da graduação em enfermagem, tempo de serviço no mínimo de um ano e área de atuação nas disciplinas voltadas a assistência de enfermagem. O estudo foi aprovado pelo Comitê de Ética em Pesquisa da Universidade Estadual do Pará, com o número de parecer 3.404.734.

Ressalta-se que os critérios de inclusão foram adaptados conforme descrito por Wild e colaboradores(Wild et al., 2019). Foram incluídos apenas os juízes cujos perfis preenchiam, pelo menos, dois critérios de inclusão. Como critérios de exclusão, foram estabelecidos: docentes de outras áreas da graduação que não seja enfermeiro e, não tenha especialização ou sem comunicação com os pesquisadores, após tentativas de contato. Os juízes foram convidados a participar da pesquisa, por meio de uma carta-convite enviada por e-mail (pessoal ou institucional). Aos juízes que aceitaram, foi solicitado enviado para o email o instrumento de validação. Foi disponibilizado aos participantes um prazo máximo de 30 dias para a devolução, a contar do envio do material. Foram convidados 17 juízes. 12 aceitaram participar da pesquisa. 
Os juízes especialistas tiveram a função de julgar o livro quanto ao conteúdo, por meio dos seguintes indicadores: objetivos (que se deseja atingir com a utilização do livro); estrutura e apresentação das orientações e relevância (características do grau de significação do material apresentado). Foi disponibilizado um espaço para sugestões.

As questões do instrumento foram divididas em blocos. Os participantes responderam, considerando as pontuações adotadas para julgamento, optando pelas seguintes respostas: (1) Discordo totalmente ;(2) Discordo; (3) Nem discordo/nem concordo; (4) Concordo; (5) Concordo totalmente Caso a resposta fosse discordo totalmente, discordo ou nem discordo/nem concordo, os juízes teriam que inserir um parecer descritivo argumentativo para justificar a resposta.

Para a análise quantitativa dos dados, foi realizado o cálculo do Índice de Validade de Conteúdo (IVC). O IVC mede a proporção dos juízes que estão em concordância sobre determinado aspecto do instrumento e de seus itens (Santos et al., 2020). Esse método emprega a utilização de escala tipo Likert, com pontuação de um a cinco. O escore do índice é calculado, por meio da soma de concordância dos itens que foram assinalados com a opção "1" e "2" dividida pelo número total de respostas (10). Em relação às médias obtidas, os itens deveriam alcançar IVC maior ou igual a 0,70 (70\%), adotado como critério de decisão sobre a pertinência e a aceitação de cada item (Alexandre, \& Coluci, 2011).

Para avaliar o livro como um todo, foi realizado o cálculo de IVC global que consistiu na somatória de todos os IVC calculados separadamente e dividido pelo número de itens do instrumento (11). Os dados foram compilados e analisados, utilizando-se o programa Statistical Package for the Social Sciences (SPSS), versão17.0. Os mesmos foram tratados com base na análise estatística descritiva, com distribuição de frequência simples.

Para avaliar o questionário usado nesse estudo foi utilizado o coeficiente de alfa de Cronbach, esse coeficiente é uma ferramenta estatística bastante relevante e uma das mais utilizadas, no que tange os estudos sobre a construção de testes e suas aplicações. Essa ferramenta se mostrou válida, uma vez que indica uma média razoável de confiabilidade em uma só aplicação, por sua fórmula geral de coeficiente permitir que ele seja usado por vários formatos de testes e por poder ser facilmente calculado por princípios estatísticos básicos (Espinoza \& Muñoz, 2018).

Tais instrumentos metodológicos foram relatados como válidos no processo de validação de conteúdo citado nos artigos de validação de tecnologias educativas, manuais, livros, cartilhas e materiais de apoio aos profissionais de saúde e/ou acadêmicos. Servindo como suporte para a conduta clínica, terapêutica e de educação permanente (De Carvalho Oliveira et al, 2021)

\section{Resultados e Discussão}

A amostra foi composta por 12 juízes enfermeiros que foram caracterizados de acordo com a faixa etária, tempo de formação, área de trabalho, titulação e área de atuação. A caracterização do perfil de juízes da amostra está representada no Quadro 1. 
Research, Society and Development, v. 10, n. 13, e162101320935, 2021

(CC BY 4.0) | ISSN 2525-3409 | DOI: http://dx.doi.org/10.33448/rsd-v10i13.20935

Quadro 1 - Caracterização do perfil de juízes.

\begin{tabular}{|c|c|c|}
\hline Perfil dos juízes & $\begin{array}{c}\text { Frequência } \\
\text { Absoluta }\end{array}$ & $\begin{array}{l}\text { Frequência } \\
\text { Relativa \% }\end{array}$ \\
\hline \multicolumn{3}{|c|}{ Faixa etária (anos) } \\
\hline$<30$ & 2 & 16,7 \\
\hline 30 a 34 & 6 & 50 \\
\hline$>=35$ & 4 & 33,3 \\
\hline \multicolumn{3}{|c|}{ Tempo de formação (anos) } \\
\hline 01 a 05 & 3 & 25 \\
\hline 06 a 10 & 3 & 25 \\
\hline Acima de 10 & 6 & 50 \\
\hline \multicolumn{3}{|c|}{ Área de trabalho } \\
\hline Assistência & 8 & 66,7 \\
\hline Docência & 5 & 41,7 \\
\hline Gestão & 2 & 16,7 \\
\hline \multicolumn{3}{|c|}{ Titulação } \\
\hline Especialização & 7 & 58,4 \\
\hline Mestrado & 4 & 33,3 \\
\hline Doutorado & 1 & 8,3 \\
\hline \multicolumn{3}{|c|}{ Área de atuação } \\
\hline Enfermagem em clínica & 5 & 41,7 \\
\hline Educação e ensino & 4 & 33,3 \\
\hline Urgência e Emergência & 3 & 25 \\
\hline
\end{tabular}

Fonte: Autores (2021).

O Quadro 2 representa a concordância dos itens avaliados dos domínios "Objetivos", "Estrutura e Apresentação" e "Relevância" pelos juízes, sendo que todos concordaram com a coerência e finalidade do material. 
Quadro 2 - Avaliação do livro quanto à concordância dos juízes aos objetivos, estrutura e apresentação e relevância.

\begin{tabular}{|c|c|c|c|}
\hline Domínios & Itens avaliados & $\begin{array}{c}\text { Concordo } \\
\%\end{array}$ & $\begin{array}{l}\text { Concordo } \\
\text { totalmente \% }\end{array}$ \\
\hline \multirow{4}{*}{ Objetivos } & Coerentes com as necessidades dos estudantes. & 25 & 75 \\
\hline & $\begin{array}{l}\text { Coerentes com do ponto de vista da assistência ao } \\
\text { portador de feridas. }\end{array}$ & 33,3 & 66,7 \\
\hline & $\begin{array}{l}\text { Pode circular no meio científico na área de } \\
\text { enfermagem. }\end{array}$ & 8,3 & 91,7 \\
\hline & $\begin{array}{l}\text { Atende aos objetivos da formação em assistência } \\
\text { de enfermagem. }\end{array}$ & 8,3 & 91,7 \\
\hline \multirow{9}{*}{$\begin{array}{l}\text { Estrutura e } \\
\text { Apresentação }\end{array}$} & $\begin{array}{l}\text { Suporte de orientação aos estudantes da graduação } \\
\text { em enfermagem. }\end{array}$ & 16,7 & 83,3 \\
\hline & $\begin{array}{l}\text { Mensagens apresentadas de maneira clara e } \\
\text { objetiva. }\end{array}$ & 25 & 75 \\
\hline & $\begin{array}{l}\text { As informações apresentadas estão cientificamente } \\
\text { corretas. }\end{array}$ & 25 & 75 \\
\hline & Apresenta sequência lógica do conteúdo proposto. & 16,7 & 83,3 \\
\hline & $\begin{array}{l}\text { Informações estão bem estruturadas em } \\
\text { concordância e ortografia. }\end{array}$ & 25 & 75 \\
\hline & $\begin{array}{l}\text { Informações da capa, contracapa e apresentação } \\
\text { são coerentes. }\end{array}$ & 8,3 & 91,7 \\
\hline & Tamanho do título e dos tópicos adequados. & 16,7 & 83,3 \\
\hline & O número de páginas está adequado. & 25 & 75 \\
\hline & As figuras são expressivas e suficientes. & 25 & 75 \\
\hline \multirow{4}{*}{ Relevância } & $\begin{array}{l}\text { Os temas retratam aspectos-chave que devem ser } \\
\text { reforçados. }\end{array}$ & 25 & 75 \\
\hline & $\begin{array}{l}\text { Propõe conhecimento quanto às condutas } \\
\text { adequadas durante a avaliação ao portador de } \\
\text { feridas. }\end{array}$ & 8,3 & 91,7 \\
\hline & $\begin{array}{l}\text { Aborda assuntos necessários a serem oferecidos } \\
\text { aos portadores de feridas. }\end{array}$ & 25 & 75 \\
\hline & $\begin{array}{l}\text { Adequado para ser usado por estudantes de } \\
\text { enfermagem. }\end{array}$ & 8,3 & 91,7 \\
\hline
\end{tabular}

Fonte: Autores (2021).

O Quadro 3 demonstra a pontuação e percentual de aprovação do livro de acordo com os itens dos "Objetivos", "Estrutura e apresentação" e "Relevância"; a concordância entre os juízes em relação ao material educativo obteve coeficiente alfa de Cronbach de 0,95; considerando-se excelente. Já o Quadro 4, demonstra a pontuação geral e percentual médio de aprovação de cada domínio; além disso, está representado o IVC de cada domínio e o quantitativo geral. 
Quadro 3 - Pontuação e percentual de aprovação de acordo com os itens dos Objetivos, Estrutura e apresentação e Relevância.

\begin{tabular}{|c|c|c|c|}
\hline Domínios & Itens avaliados & $\begin{array}{c}\text { Pontuação } \\
\text { Recebida }\end{array}$ & $\begin{array}{c}\% \\
\text { Aprovação }\end{array}$ \\
\hline \multirow{4}{*}{ Objetivos } & $\begin{array}{l}\text { Pode circular no meio científico na área de } \\
\text { enfermagem. }\end{array}$ & 59 & 98,3 \\
\hline & $\begin{array}{l}\text { Atende aos objetivos da formação em assistência de } \\
\text { enfermagem. }\end{array}$ & 59 & 98,3 \\
\hline & Coerentes com as necessidades dos estudantes. & 57 & 95 \\
\hline & $\begin{array}{l}\text { Coerentes com do ponto de vista da assistência ao } \\
\text { portador de feridas. }\end{array}$ & 56 & $93,3 \%$ \\
\hline \multirow{9}{*}{$\begin{array}{l}\text { Estrutura e } \\
\text { apresentação }\end{array}$} & $\begin{array}{l}\text { Informações da capa, contracapa e apresentação são } \\
\text { coerentes. }\end{array}$ & 59 & 98,3 \\
\hline & $\begin{array}{l}\text { Suporte de orientação aos estudantes da graduação } \\
\text { em enfermagem. }\end{array}$ & 58 & 96,7 \\
\hline & Sequência lógica do conteúdo proposto. & 58 & 96,7 \\
\hline & Tamanho do título e dos tópicos adequados. & 58 & 96,7 \\
\hline & Mensagens apresentadas de maneira clara e objetiva. & 57 & 95 \\
\hline & $\begin{array}{l}\text { Informações apresentadas estão cientificamente } \\
\text { corretas. }\end{array}$ & 57 & 95 \\
\hline & $\begin{array}{l}\text { Informações bem estruturadas em concordância e } \\
\text { ortografia. }\end{array}$ & 57 & 95 \\
\hline & O número de páginas está adequado. & 57 & 95 \\
\hline & As figuras são expressivas e suficientes. & 57 & 95 \\
\hline \multirow{4}{*}{ Relevância } & $\begin{array}{l}\text { Propõe conhecimento quanto às condutas adequadas } \\
\text { durante a avaliação do portador de feridas. }\end{array}$ & 59 & 98,3 \\
\hline & $\begin{array}{l}\text { Adequado para ser usado por estudantes de } \\
\text { enfermagem. }\end{array}$ & 59 & 98,3 \\
\hline & $\begin{array}{l}\text { Os temas retratam aspectos-chave que devem ser } \\
\text { reforçados. }\end{array}$ & 57 & 95 \\
\hline & $\begin{array}{l}\text { Aborda assuntos necessários a serem oferecidos aos } \\
\text { portadores de feridas. }\end{array}$ & 57 & 95 \\
\hline
\end{tabular}

Fonte: Autores (2021).

Quadro 4 - Pontuação geral, percentual médio de aprovação e IVC de acordo com os Objetivos, Estrutura e Apresentação e Relevância.

\begin{tabular}{|c|c|c|c|}
\hline $\begin{array}{c}\text { Critérios para validação do } \\
\text { manual }\end{array}$ & Pontuação Recebida & \% Aprovação & IVC \\
\hline 1. Objetivos & 231 & $96,3 \%$ & 1,00 \\
\hline 2. Estrutura e Apresentação & 518 & $95,9 \%$ & 0,98 \\
\hline 3. Relevância & 232 & $96,7 \%$ & 1,00 \\
\hline Geral & 981 & $96,2 \%$ & 0,99 \\
\hline
\end{tabular}

Fonte: Autores (2021).

Os crescentes avanços tecnológicos em saúde vêm permeando o processo de trabalho em saúde; na assistência de enfermagem, envolve um conjunto de ferramentas que podem ser cada vez mais desenvolvidas e especializadas para orientar o cuidado em saúde ao cliente. Materiais como esses propiciam uma visão ampliada de ações susceptíveis de desenvolvimento no campo da saúde, incentivando novas ideias e trabalhos voltados à temática da promoção da saúde (Benevides et al., 2016).

O Quadro 1 evidencia que os juízes eram de diferentes áreas de atuação, a saber: Enfermagem em Clínica, Educação e Ensino e Urgência e Emergência. Assim, torna-se notório a confiabilidade da validação da tecnologia educacional; haja em vista que a presença desses profissionais especialistas promove diversidade de opiniões e enfoques sobre a mesma temática, garantindo um ensino efetivo e de qualidade (Santos et al., 2020). 
A construção dos questionários seguiu ciclos sequenciais utilizando a técnica Delphi. Assim, o instrumento utilizado para validação deste material educativo em saúde enfocou três grupos de fatores relacionados ao conteúdo: Objetivos, Estrutura e Apresentação e Relevância (Bessa, 2012)

Nessa perspectiva, é importante ressaltar que os materiais educativos avaliados por instrumentos de conteúdo na área da saúde devem objetivar facilitação do trabalho dos profissionais de saúde para orientar e educar pacientes e familiares. Acrescenta-se ainda que tem a finalidade de promover a saúde, garantindo efetividade do cuidado (Leite et al., 2018).

Os profissionais especialistas avaliaram os itens de cada um desses domínios. Como demonstrado no Quadro 2, houve concordância de $100 \%$ dos juízes quanto aos itens avaliados nos domínios "Objetivos", "Estrutura e Apresentação" e "Relevância"; sendo que a maioria concordou totalmente. Isso comprova a possibilidade de utilização desse instrumento no meio acadêmico, profissional e em educação continuada.

Conforme apresentado no Quadro 3, houve excelente pontuação e percentual de aprovação; dessa forma, o domínio "Objetivos" obteve aprovação de 96,3\% pelos juízes e apresentou índice de concordância máxima com IVC de 1,0. Pode-se considerar, portanto, que o livro atendeu as finalidades propostas; podendo circular no meio científico, contribuir na formação do enfermeiro e ser coerente com as necessidades dos estudantes e do portador de feridas.

O estudo de Santos e colaboradores (2020), também sobre validação de tecnologia educacional, ressalta que esse domínio em um instrumento é necessário para o entendimento do propósito para o qual o conteúdo foi elaborado e assim ser divulgado no meio cientifico. Esse estudo também obteve excelente IVC de 0,96, avaliando itens semelhantes aos avaliados neste estudo.

O domínio "Estrutura e Apresentação" foi o que apresentou menor IVC e aprovação, porém ainda considerado excelente; demonstrando que o material se apresentava em sequência lógica, coerente, tamanho do título e dos tópicos adequados e com imagens expressivas e suficientes.

É válido destacar que mesmo havendo pontuação o suficiente para validação, apenas $75 \%$ dos especialistas concordaram totalmente de que as mensagens se apresentavam de maneira clara e objetiva ou que as informações estavam bem estruturadas em concordância e ortografia e que as figuras eram expressivas e suficientes. Diante disso, os pesquisadores revisaram o conteúdo e imagens para melhor adequação, o que garante maior credibilidade ao material produzido e representatividade.

Nesse sentido, Wild e colaboradores (2019) destacam que, embora seja um domínio de difícil finalização, o emprego apropriado de ilustrações permite a legibilidade e compreensão das mensagens apresentadas; uma vez que torna a leitura mais atrativa ao despertar e manter o interesse pela leitura, transformando as informações textuais em linguagem visual.

Ainda nessa perspectiva, levando em consideração que as figuras ocupam importante papel na comunicação ao transformar as informações textuais em linguagem visual; as fotos utilizadas de simulação em feridas no instrumento foram obtidas no próprio ambiente em que foi realizado o evento de exposição, representando o cenário real. Logo, pode-se inferir que o uso crescente de materiais educativos enquanto recursos no processo de educação em saúde criou novas possibilidades de interação entre o ensino em feridas e os acadêmicos de enfermagem.

Cruz e colaboradores (2016), ressalta que o instrumento educacional deve apresentar estrutura coerente, coesa, organizada, suficiente e boa elaboração textual; para isso, é necessário que o conteúdo mantenha o foco na temática proposta e que em seus tópicos, parágrafos ou trechos haja sequência lógica de ideias. Considera-se, assim, que o instrumento conseguiu atender os propósitos para os quais foi elaborado, sendo capaz de orientar a construção de conteúdos educativos em saúde em formatos diversos. 
O domínio Relevância apresentou aprovação de 96,7\% e excelente IVC de 1,0; havendo concordância de que a tecnologia educacional produzida propõe conhecimento quanto às condutas adequadas durante a avaliação do portador de feridas e que é adequada para ser usada por acadêmicos de enfermagem. No entanto, apenas $75 \%$ concordaram totalmente de que os temas retratavam aspectos-chave que devem ser reforçados e que abordava assuntos necessários a serem oferecidos aos portadores de feridas; assim, mesmo alcançando pontuação suficiente, foi feita uma reavaliação do instrumento.

Assim sendo, o material tem alta relevância no processo educacional e pode contribuir para uma aprendizagem mais ativa e criativa. O instrumento foi considerado validado pelos juízes, demonstrando consistência interna desejável com coeficiente alfa de Cronbach de 0,95 o qual se trata de um importante instrumento quando se objetiva medir construto único por meio de múltiplos itens.

O livro apresentou IVC global de 0,99 (quadro 4) com excelente nível de concordância, podendo-se inferir que a ferramenta é representativa do conteúdo a ser abordado sobre o ensino de enfermagem em relação à ferida. Resultado semelhante a esse, foi encontrado no estudo de Benevides e colaboradores (2016) com IVC global de 0,97.

Logo, pode-se afirmar que o material elaborado contribui no processo de ensino-aprendizagem no tratamento de feridas, sendo um importante instrumento para melhoria do acesso da população a tratamentos que sejam comprovadamente eficazes. Além disso, ressalta-se que a técnica de simulação realística de feridas é de extrema importância para capacitação dos profissionais, pincipalmente dentro do processo de ensino na graduação de enfermagem, pois desenvolve habilidades e competências para prestar assistência a esse paciente (Joaquim, \& Silvino, ZR 2020)

Evidencia-se ainda que, com a validação da tecnologia, o livro pode ser utilizado como literatura no ensino e tratamento de feridas, o que pode repercutir na melhoria da pratica profissional do enfermeiro, tendo em vista seu papel fundamental na avaliação do cliente portador de feridas, prescrição do cuidado mais adequado e supervisão da equipe de enfermagem na realização do curativo (Macedo et al., 2019).

\section{Considerações Finais}

Diante do exposto, conclui-se que esse estudo alcançou o seu objetivo, uma vez que, após o estudo, o livro, foi considerado pelos juízes especialistas como um material educativo valido com IVC geral de 0,99 e em relação a confiabilidade o coeficiente alfa de Cronbach demostrou uma consistência interna de 0,95, configurando-se em uma tecnologia educacional válida para ser usada no ensino de avaliação e tratamento de feridas no ensino de enfermagem, a fim de proporcionar um processo de aprendizagem significativo e efetivo para os discentes.

As diversidades de áreas de atuação dos juízes conferem ao livro relevância e credibilidade. Sendo assim, o uso de tal tecnologia é de extrema relevância para o meio científico, repercutindo em uma assistência integral ao paciente. Através desta pesquisa, espera-se que os profissionais da saúde tenham mais interesse sobre a temática e realizem novos estudos sobre o uso de tecnologias educacionais aplicadas processo educacionais de futuros profissionais da saúde, preenchendo lacunas que possa ter sido criado em seu processo de formação.

Sugerimos que após a elaboração de instrumentos de ensino para acadêmicos ou materiais de educação permanente para profissionais, tais como manuais, livros didáticos educacionais, cartilhas e cadernetas o rigor cientifico do processo de validação dando credibilidade, aplicabilidade e relevância sejam respeitados. Recomendados que na realização do processo de validação através da técnica de Delphi, Alfa de Cronbach para avaliação dos questionários e respostas dos juízes especialistas, índice de validade de conteúdo IVC estruturem uma técnica rigorosa construindo e efetivando assim a validade dos materiais acadêmicos ou de apoio profissional como válido e utilizável de forma sistemática e confiável. 


\section{Referências}

Benevides, J. L., Coutinho, J. F. V., Pascoal, L. C., Joventino, E. S., Martins, M. C., Gubert, F. do A., \& Alves, A. M. (2016). Development and validation of educational technology for venous ulcer care. Revista Da Escola de Enfermagem, 50(2), 306-312. https://doi.org/10.1590/S0080-623420160000200018

Capella, S. O., Tillmann, M. T., Félix, A. O. C., Fontoura, E. G., Fernandes, C. G., Freitag, R. A., Santos, M. A. Z., Félix, S. R., \& Nobre, M. O. (2016). Potencial cicatricial da Bixa orellana L. em feridas cutâneas: Estudo em modelo experimental. Arq. Bras. Med. Vet. Zootec., 68(1), 104-112. https://doi.org/10.1590/1678-4162-8374

Carvalho, D. S. de, Silva, A. G. I. da, Ferreira, S. R. M., \& Braga, L. C. (2019). Construção de tecnologia educacional para estomizados: enfoque no cuidado da pele periestoma. Rev. Bras. Enferm., 72(2), 427-434. https://doi.org/10.1590/0034-7167-2016-0024

Cruz, F. O. D. A. M. Da., Ferreira, E. B., Vasques, C. I., Mata, L. R. F. Da, \& Reis, P. E. D. Dos. (2016). Validation of an educative manual for patients with head and neck cancer submitted to radiation therapy. Revista Latino-Americana de Enfermagem, 24. https://doi.org/10.1590/1518-8345.0949.2706

de Carvalho Oliveira, G. R., Percário, S., Botelho, N. M., de Andrade, M. C., de Oliveira, J. M., da Silva Alexandre, A. D., \& dos Santos Junior, H. S. (2021). Validação de uma tecnologia: manual de acolhimento ambulatorial e cirúrgico na diversidade sexual. Research, Society and Development, 10(8), e32310817467-e32310817467.

Espinoza, S. C., \& Muñoz, F. N. (2018). Ventajas del alfa ordinal respecto al alfa de Cronbach ilustradas con la encuesta AUDIT-OMS. Revista Panamericana de Salud Pública, 42, e65. https://doi.org/10.26633/RPSP.2018.65

Gonçalves, M. B. B., Rabeh, S. A. N., \& Terçariol, C. A. S. (2015). Contribuição da educação a distância para o conhecimento de docentes de enfermagem sobre avaliação de feridas crônicas. Rev. Latino-Am. Enfermagem, 23(1), 122-129. https://doi.org/10.1590/0104-1169.3606.2533

Kumakura, A. R. de S. O., Silva, J. L. G., \& Gonçalves, N. (2018). Da aula teórica ao uso da simulação para ensinar o cuidar de pessoas com queimaduras: relato de caso. Escola Anna Nery, 22(3), 2018. https://doi.org/10.1590/2177-9465-EAN-2017-0391

Leite, S. de S., Áfio, A. C. E., Carvalho, L. V. de, Silva, J. M. da, Almeida, P. C. de, \& Pagliuca, L. M. F. (2018). Construction and validation of an Educational Content Validation Instrument in Health. Revista Brasileira de Enfermagem, 71, 1635-1641. https://doi.org/10.1590/0034-7167-2017-0648

Lima, B. R. de;, Silva, G. O., \& Galdino-Júnior, H. (2020). Avaliação das prescrições de enfermagem e médicas para curativos em um hospital universitário: implicações éticas . Enferm. Foco (Brasília), 11(5), 67-72. https://pesquisa.bvsalud.org/portal/resource/pt/biblio-1177216

Macedo, E. A. B. de, Freitas, C. C. S. de, Dionisio, A. J., \& Torres, G. de V. (2019). Knowledge of the care of wounded patients: evidence of validity of an instrument. Revista Brasileira de Enfermagem, 72(6), 1562-1570. https://doi.org/10.1590/0034-7167-2018-0643

Mazzo, A., Miranda, F. B. G., Meska, M. H. G., Bianchini, A., Bernardes, R. M., \& Pereira, G. A. (2017). Ensino de prevenção e tratamento de lesão por pressão utilizando simulação. Escola Anna Nery, 22(1), 2018. https://doi.org/10.1590/2177-9465-EAN-2017-0182

Melo, L. H. de A., Bernardo, T. H. L., Macedo, J. K. S. dos S., Francisco, L. C. F. de L., \& Barros, A. C. (2020). Aplicacao da teoria de Orem no ambito das feridas: uma revisao integrativa. ESTIMA, Braz. J. Enterostomal Ther, 18(MELO, L. H. DE A. et al. Aplicacao da teoria de Orem no ambito das feridas: uma revisao integrativa. ESTIMA, Braz. J. Enterostomal Ther, v. 18, 2020.). https://www.revistaestima.com.br/index.php/estima/article/view/821/pdf_1

Salome, G. M. (2020). Desenvolvimento de um material educativo para a prevencao e o tratamento das lesoes por friccao. STIMA, Braz. J. Enterostomal Ther, 18. https://www.revistaestima.com.br/estima/article/view/923/371

Santos, A. da S., Rodrigues, L. do N., Andrade, K. C., Santos, M. S. N. Dos, Viana, M. C. A., \& Chaves, E. M. C. (2020). Construção e validação de tecnologia educacional para vínculo mãe-filho na unidade de terapia intensiva neonatal. Rev. Bras. Enferm., 73(4), e20190083. https://doi.org/10.1590/00347167-2019-0083

Silva, J. L. G., \& Oliveira-Kumakura, A. R. de S. (2018). Simulação clínica para ensino da assistência ao paciente com ferida . Rev Bras Enferm [Internet], 71(4), 1890-1895. https://doi.org/10.1590/0034-7167-2017-0170

Wild, C. F., Nietsche, E. A., Salbego, C., Teixeira, E., \& Favero, N. B. (2019). Validação de cartilha educativa: uma tecnologia educacional na prevenção da dengue. Rev. Bras. Enferm., 72(5), 1318-1325. https://doi.org/10.1590/0034-7167-2018-0771

Joaquim, F. L., \& Silvino, Z. R (2020). Validação de tecnologia gerencial para o cuidado de pacientes com úlceras venosas crônicas, atendidos em ambulatórios no contexto hospitalar. Research, Society and Development, 9 (8), e103985256-e103985256.

Bessa, M. E. P. (2012). Elaboração e validação de conteúdo do protocolo de Intervenções de enfermagem para idosos com risco fragilidade.

Alexandre, N. M. C., \& Coluci, M. Z. O. (2011). Validade de conteúdo nos processos de construção e adaptação de instrumentos de medidas. Ciência \& Saúde Coletiva, 16, 3061-3068. 